\title{
Assessing shot-peening residual stresses by using the incremental hole-drilling technique and laser interferometry (DSPI)
}

\author{
J.P. Nobre ${ }^{1,2, a^{*}}$, M. Oliveira ${ }^{1, b, \S}, A$. Albertazzi $^{3, c}$, M. Viotti ${ }^{4, d}$, A.C. Batista ${ }^{5, e}$, \\ L. Coelho ${ }^{1,4, f}$, M.J. Marques ${ }^{1,5,9}$ \\ ${ }^{1}$ CEMDRX, Department of Physics, University of Coimbra, 3004-516 Coimbra, Portugal
}

${ }^{2}$ School of Mechanical, Industrial and Aeronautical Engineering, University of the Witwatersrand, WITS 2050, Johannesburg, South Africa

${ }^{3}$ Labmetro, Federal University of Santa Catarina (UFSC), 88040-900 Florianópolis, Brasil

${ }^{4}$ School of Technology and Management, Polytechnic Institute of Leiria, 2411-901Leiria, Portugal

${ }^{5}$ Department of Physics Engineering, University of Porto, 4200-465 Porto, Portugal

a Joao.Nobre@wits.ac.za, ${ }^{b}$ miguel.lousada@gmail.com, ${ }^{c}$ albertazzi@labmetro.ufsc.br, d matiasviotti@gmail.com, ${ }^{\mathrm{e}}$ castanhola@fis.uc.pt, ${ }^{\dagger}$ luis.coelho@ipleiria.pt, ${ }^{9}$ mjvaz@fe.up.pt

* Corresponding author

Keywords: shot-peening, residual stresses, hole-drilling method, laser interferometry, digital speckle pattern interferometry (DSPI).

Abstract. The incremental hole-drilling technique was applied to determine residual stress profiles in shot-peened steel layers. The accuracy of using an enhanced Digital Speckle Pattern Interferometry technique for measuring the strain relaxation arising around the drilled holes and, consequently, the in-depth residual stress distribution induced by shot-peening, was evaluated. The experimental results were systematically compared with those determined using standard electric strain-gauges. The X-ray diffraction technique was chosen as reference due to its high accuracy to determine shot-peening residual stresses.

\section{Introduction}

The aim of this investigation was to perform a comparative analysis between two different techniques for measuring deformations, strain gauges and laser interferometry, to be applied with the incremental hole-drilling technique (IHD) to determine non-uniform residual stresses. This investigation was made through a partnership between the CFisUC of the University of Coimbra, Portugal, and the Labmetro of the Federal University of Santa Catarina, Brazil. In order to perform this analysis, several experiments were made using both strain gauges and, afterwards, a laser interferometry system based on the Digital Speckle Pattern Interferometry (DSPI) technique developed and patented by A. Albertazzi and his team of the Labmetro [1].

The tests were performed on two steel samples, with different mechanical properties, and its results were compared. These results were also compared with referential values provided by the Xray diffraction technique, combined with the electrolytic layer removal technique.

After the analysis of the experimental processes and its results, it was possible to conclude that DSPI is a technique that has several advantages in terms of the experimental process against the traditional strain gauge method, especially if done through a compact system like the one developed by Albertazzi et al. [2]. The main advantages are the faster test time and the easy preparation of the test surfaces. When those advantages are combined with the fact that the system is compact and portable, it allows for easier and faster component testing outside of the laboratory environment. Nevertheless, the experimental results were not as expected. The DSPI measurements were very far off from, and more inconsistent, than the results obtained by the strain gauges and the X-ray diffraction techniques. It was concluded that, due to the fact that the application of DSPI in the hole drilling method is still a relatively new method, the mathematical models necessary to adapt this technique to the measurement of deformations around a hole should still to be developed, once this technique is not yet able to provide reliable results in all situations, as it was the case of this work's experimental results. 


\section{Incremental Hole-Drilling Technique using Strain Gauge Technology}

The incremental hole-drilling technique basically consists of machining a small hole (typically 1 to $5 \mathrm{~mm}$ ), step by step, in the test material's surface and measuring the surface strain relaxation induced by each incremental depth. Specific calibration functions are needed, since it is necessary to correlate the strain relaxation measured at the material's surface with the average stress existing in each depth increment. These coefficients can be determined through a numerical calibration by using the finite element method (FEM). For linear elastic and isotropic materials, these coefficients can be almost considered material independent and, therefore, normalization for a given strain gauge rosette and hole geometry is possible. ASTM E 837 American standard has normalized strain gauge rosettes (type $\mathrm{A}, \mathrm{B}$ and $\mathrm{C}$ ), presenting the correspondent calibration coefficients to be used for calculation of in-depth non-uniform residual stresses by using the so-called integral method [3, 4].

The integral method considers that the strain relief at the material's surface is the accumulated result of the residual stresses originally existing in the zone of each successive increment, throughout the total hole depth. The integral method identifies the individual contribution of the stress in each increment to the total relieved strain measured at the material's surface. The stress is considered constant for each incremental depth and equal to the mean value of the real profile in that depth increment. The corresponding total strain can be divided in three different components. The hydrostatic strain component $p_{i}$ and the shear strain components $q_{i}$ and $t_{i}$, measured after i depth increments, can be found from the sum of strains due to the residual stresses relaxation that have occurred in each depth increment prior to hole drilling, as [3, 4]:

$$
\left\{\begin{aligned}
p_{i} & =\frac{1+v}{E} \sum \bar{a}_{i j} P_{j} \\
q_{i} & =\frac{1}{E} \sum \bar{b}_{i j} Q_{j} \quad 1 \leq j \leq i, \\
t_{i} & =\frac{1}{E} \sum \bar{b}_{i j} T_{j}
\end{aligned}\right.
$$

where $\mathrm{E}$ is the Young modulus, $\mathrm{v}$ the Poisson's coefficient and $\mathrm{p}, \mathrm{q}$ and $\mathrm{t}$, are related with the measured strain, by each strain gauge in the normalized rosette, by:

$$
\left\{\begin{array}{c}
p=\frac{\varepsilon_{3}+\varepsilon_{1}}{2} \\
q=\frac{\varepsilon_{3}-\varepsilon_{1}}{2}, \\
t=\frac{\varepsilon_{3}+\varepsilon_{1}-2 \varepsilon_{2}}{2}
\end{array}\right.
$$

and $\mathrm{P}, \mathrm{Q}$ and $\mathrm{T}$, the corresponding transformed stresses, are related with the normal stress components in the directions of gauges 1 and $3, \sigma_{1}$ and $\sigma_{3}$, respectively, and the shear stress component normal to directions 1 and $3, \tau_{13}$ :

$$
\left\{\begin{array}{c}
P=\frac{\sigma_{3}+\sigma_{1}}{2} \\
Q=\frac{\sigma_{3}-\sigma_{1}}{2} \\
T=\tau_{13}
\end{array}\right.
$$


The principal stresses $\sigma_{\max }, \sigma_{\min }$ and their orientation $\beta$ (with reference to the strain gauge 1) will be given by:

$$
\left\{\begin{array}{c}
\sigma_{\max }=P+\sqrt{Q^{2}+T^{2}} \\
\sigma_{\min }=P-\sqrt{Q^{2}+T^{2}} \\
\beta=\frac{1}{2} \arctan \frac{-T}{-Q}
\end{array}\right.
$$

$\bar{a}_{i j}$ and $\bar{b}_{i j}$ in equations (1) are called calibration coefficients, being material independent, can be numerically determined and normalized for ASTM strain gauges rosettes. The calibration coefficients matrices are lower triangular. Their values depend on the position of the stressed depth increment $j$ and the actual hole depth $i$, and can only be determined by means of a numerical calibration. After $P, Q$ and $T$ are determined; equation (4) can be used to determine the principal stresses and their orientation for each depth increment. It should be noted that small errors in the measured strains and hole depths can lead to important errors in residual stress results. These errors increase with increasing hole depth. For this reason the maximum evaluation depth is limited to about 0.3 to 0.4 of the mean radius of the strain gauge rosette. Furthermore, for a given hole, the error increases with the increase in the number of depth increments [9].

\section{Incremental Hole-Drilling Technique using DSPI Technology}

The principles presenting above, using strain gauge technology, can be applied if the surface strain relaxation is measured using laser interferometry.

Fig. 1 shows the configuration of the DSPI radial in-plane interferometer. Expanded light from a $660 \mathrm{~nm}$ wavelength diode laser (L) passes through a central elliptical hole of mirror M1 and illuminates mirrors $\mathrm{M}_{2}$ and $\mathrm{M}_{3}$. After that, laser beam is reflected back to mirror $\mathrm{M}_{1}$ being directed to the collimating lens (CL) to obtain an annular parallel beam. The central elliptical hole located at mirror $\mathrm{M}_{1}$ has three main functions: (a) to make possible that light coming from the laser source illuminates mirrors $\mathrm{M}_{2}$ and $\mathrm{M}_{3}$, (b) to avoid that laser light reaches directly the specimen surface having triple illumination and (c) to make available a viewing window for the digital camera. Finally, the collimated light reaches a special diffractive optical element (DOE) formed by a set of circular diffracting lines with a regular spacing of about $1.3 \mu \mathrm{m}$. The first diffracted order is deflected towards a central circular area on the specimen and produces a double illuminated circular area of about $8 \mathrm{~mm}$ in diameter with radial sensitivity.
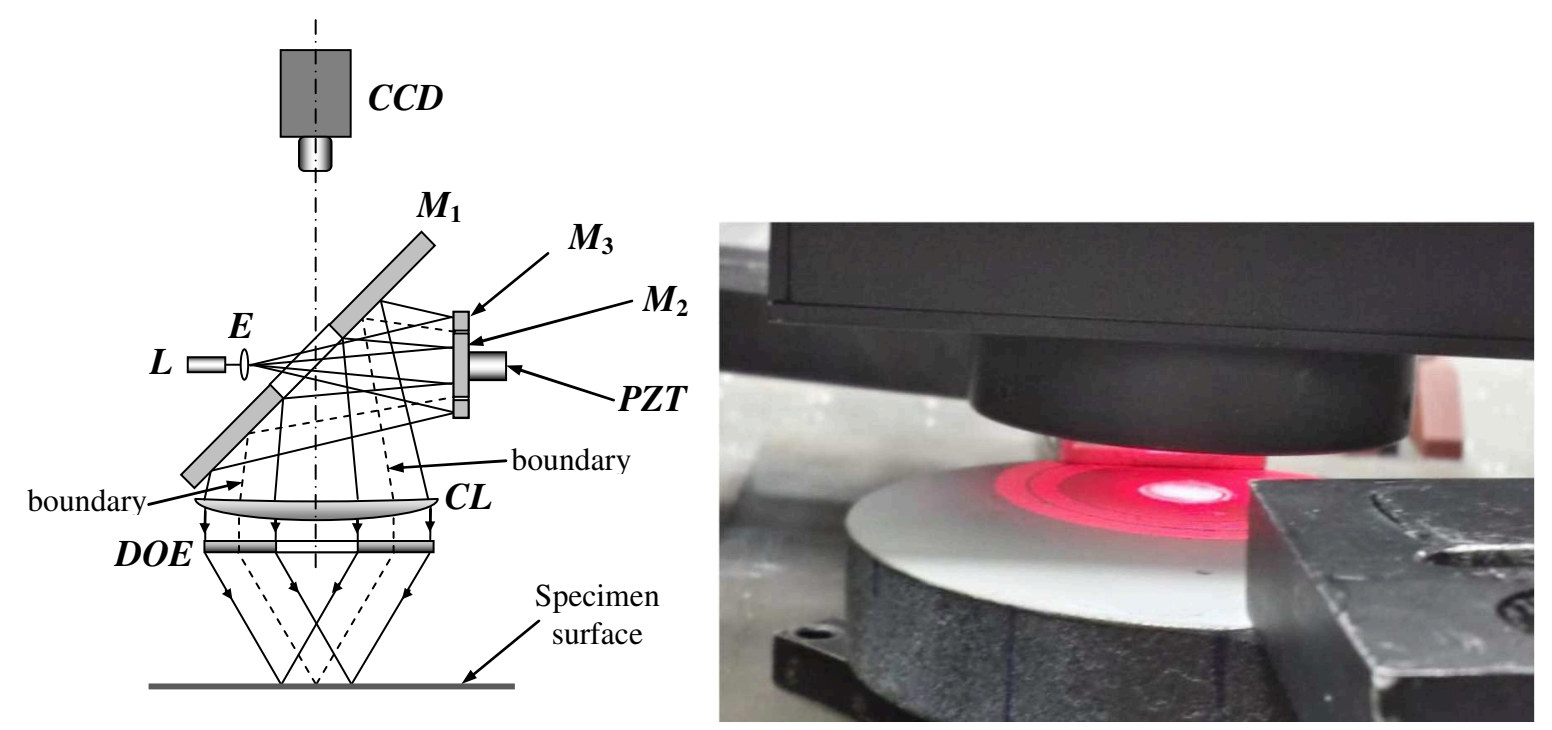

Fig. 1 Optical arrangement of the radial in-plane interferometer (left) and a DSPI measuring example (right) 
Mirror $\mathrm{M}_{2}$ is a mobile mirror since it is joined to a piezoelectric actuator (PZT). On the contrary, mirror $\mathrm{M}_{3}$ is fixed and it has a circular hole with a diameter slightly larger than $\mathrm{M}_{2}$ diameter. PZT actuator linearly moves $\mathrm{M}_{2}$ along its axial axis generating a relative phase difference between beams reflected by $\mathrm{M}_{2}$ (central beam) and $\mathrm{M}_{3}$ (external one). Dashed lines in Fig. 1 (left) show boundaries between them. Every point laying in the specimen surface is double illuminated with one light ray coming from $\mathrm{M}_{2}$ and the other from $\mathrm{M}_{3}$. Thus, PZT enables the introduction of a constant phase shift between both rays in order to calculate wrapped phase maps using phase shifting algorithms.

In order to measure residual stresses, this radial DSPI configuration was implemented in a portable system [2] that is also equipped with an ultra-high speed air turbine to drill blind holes. In order to measure residual stresses the following procedure is used. The system is initially positioned over the surface to be measured and stiffly fixed using three legs with sharp tips and a set of rare earth magnets. Afterwards, a set of five $90^{\circ}$ phase-shifted speckle interferograms is acquired and the reference phase distribution is computed and stored in the computer. Then, the drilling unit is activated and used to drill a $1.6 \mathrm{~mm}$ diameter blind hole with a depth of about only $\mathrm{h}_{1}=0.050 \mathrm{~mm}$. After waiting a few seconds, a new set of five phase-shifted speckle interferograms is acquired and its corresponding phase distribution is calculated and stored. Another drilling step is performed increasing the hole depth of about $0.050 \mathrm{~mm}$ each time $\left(\mathrm{h}_{i+1}=\mathrm{h}_{\mathrm{i}}+0.050 \mathrm{~mm}\right)$ and another set of five phase-shifted interferograms is acquired and phase distribution computed. The process is repeated up to the drilled hole depth reaches $1.00 \mathrm{~mm}$. The phase difference increments are computed for each depth increment. Each phase increment is digitally processed and the corresponding parameters are extracted from the phase difference images.

Fig. 2 shows a typical phase difference image. The fringe pattern is influenced by stress relaxation produced by the blind hole increment, as well by a small amount of rigid-body translation. The rigid body component is mathematically eliminated by computing the symmetrical part of the image. That is done simply by adding phase values $(\phi)$ for each diametrically opposed pixel pairs: $\phi(r, \theta)+\phi(r, \theta+\pi)$ and assigning the results to both pixels. Fig. 3 shows the resulting symmetrical image for Fig. 2.

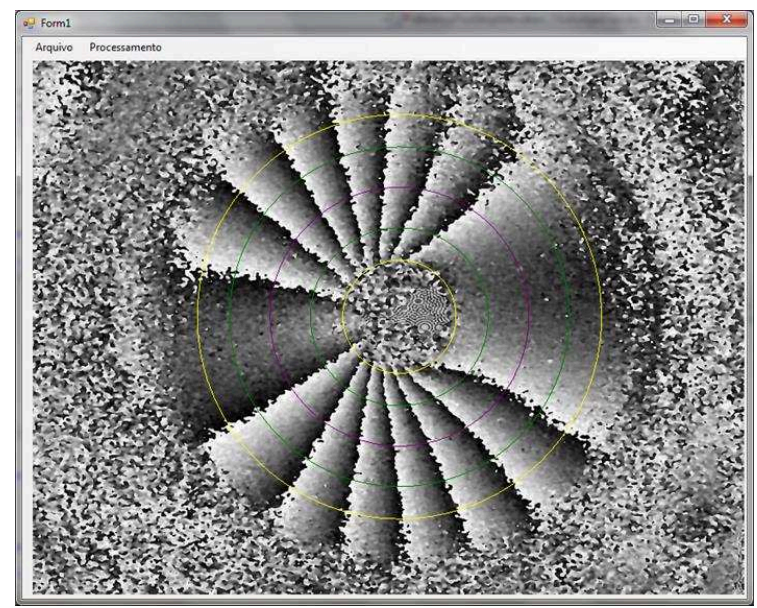

Fig. 2 A typical complete phase difference image

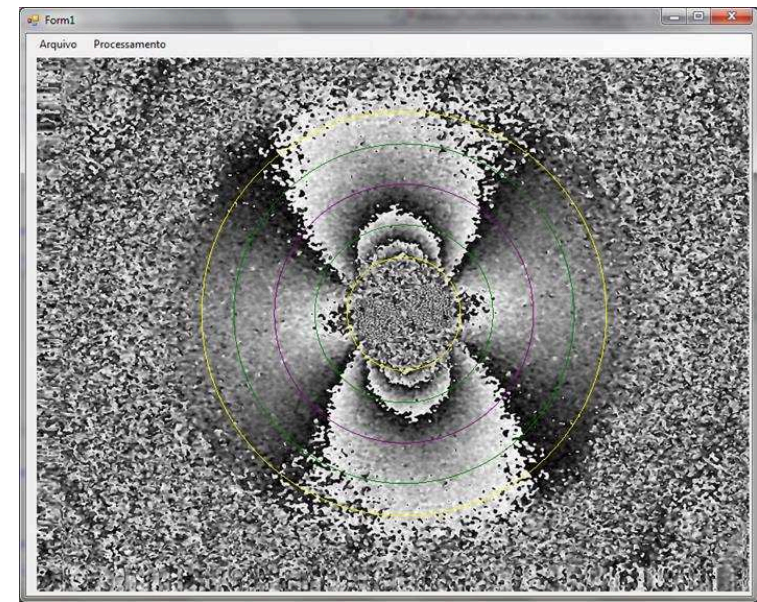

Fig. 3 Symmetrical part of Fig. 2

The next processing step is phase unwrapping of the fringe pattern. Fig. 4 shows the resulting image. Note that only data in the region between the inner and external circles are used. That region shows a quite smooth texture. A set of about 100 to 150 thousand data points is extracted from that region and the radial displacement is computed and fitted to an appropriate parametric mathematical model. Fig. 5 shows the overlapping of both experimental fringe pattern and fitted model. 


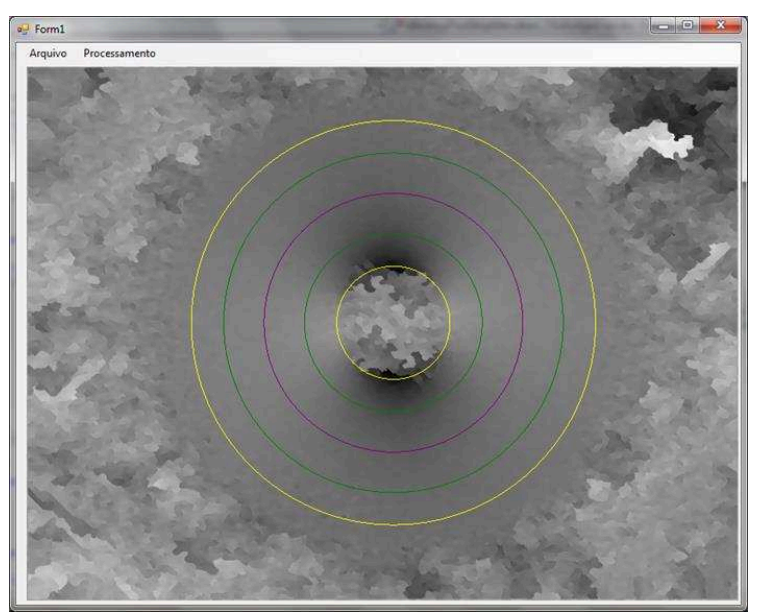

Fig. 4 Unwrapped version of Fig. 3

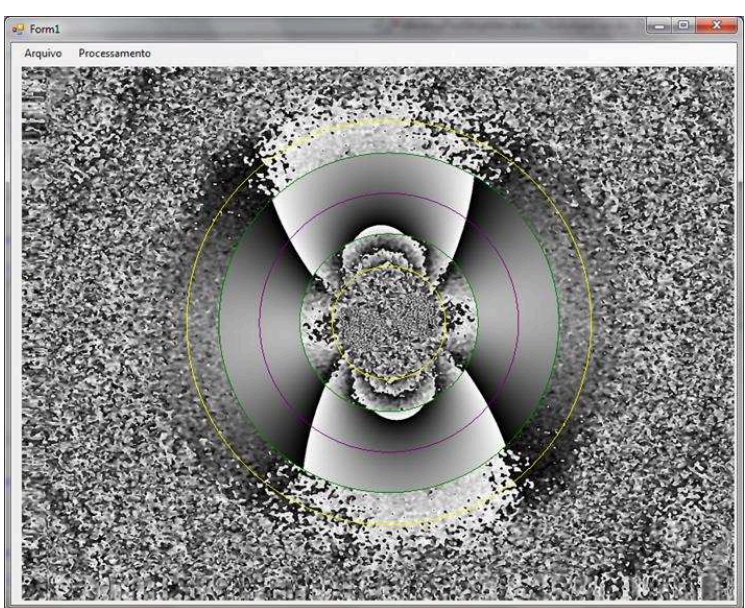

Fig. 5 Experimental and fitted fringe patterns

The radial displacement field is numerically fitted to the equation (9). The variables $r$ and $\theta$ are polar coordinates; $r_{0}$ is the blind hole radius; $v$ is the Poisson ratio. The quantity $\varepsilon_{S \mathrm{i}}$ is the equivalent principal residual strain sum $\left(\varepsilon_{\mathrm{Si}}=\varepsilon_{1 \mathrm{Ri}}+\varepsilon_{2 \mathrm{Ri}}\right)$ and $\varepsilon_{\mathrm{Di}}$ is the equivalent principal strains difference $\left(\varepsilon_{D i}=\varepsilon_{1 R i}-\varepsilon_{2 R i}\right)$ for the layer " $i$ " and $\beta_{i}$ is the principal direction angle for this same layer.

$$
u_{r}(r, \theta)=\frac{1+v}{1-v} \frac{r_{0}}{r} r_{0} \varepsilon_{S i}+\left[\frac{4}{1+v} \frac{r_{0}}{r}-\left(\frac{r_{0}}{r}\right)^{3}\right] r_{0} \varepsilon_{D i} \cos \left(2 \theta-2 \beta_{i}\right)
$$

The principal residual strains sum and difference $\varepsilon_{S i}$ and $\varepsilon_{D i}$ computed from Eq. (9) are equivalent to the strains of an infinite plate with a through hole on it. In order to compute the residual stresses gradients in each material layer as a function of the depth $\mathrm{h}$ the integral method [4] is used. The ASTM E 837-13a [3] standard has a set of relaxation coefficients given as triangular tables. They are prepared for strain gauges measurement with a given geometry. In order to use DSPI, data normalization has to be done in such coefficients in order to make the coefficients coincides with the amount of relived stress in a scale from 0 to 1.0. The normalization is done for each $a_{i j}$ and $b_{i j}$ coefficients for type A rosette by a simple multiplication given in Eq. (10). The minus signals are necessary since Eq. (9) is expressed in terms of displacements and not as strains, as in the ASTM E 837 standard [3].

$$
a_{n i j}=-5,3338 a_{i j} \quad \text { and } \quad b_{n i j}=-2,13291 b_{i j}
$$

Once the quantities $\varepsilon_{\mathrm{Si}}$ and $\varepsilon_{\mathrm{Di}}$ are computed for each layer with known depth $\mathrm{h}_{\mathrm{i}}$, the residual stresses can be computed solving the system of Eqs. (11) and (12):

$$
\begin{gathered}
\frac{1-v}{E}\left[\begin{array}{ccc}
a n_{11} & 0 & 0 \\
a n_{21} & a n_{22} & 0 \\
a n_{31} & a n_{32} & a n_{33}
\end{array}\right]\left\{\begin{array}{l}
\sigma_{S 1} \\
\sigma_{S 2} \\
\sigma_{S 3}
\end{array}\right\}=\left\{\begin{array}{l}
\varepsilon_{S 1} \\
\varepsilon_{S 2} \\
\varepsilon_{S 3}
\end{array}\right\} \\
\frac{1+v}{E}\left[\begin{array}{ccc}
b n_{11} & 0 & 0 \\
b n_{21} & b n_{22} & 0 \\
b n_{31} & b n_{32} & b n_{33}
\end{array}\right]\left\{\begin{array}{l}
\sigma_{D 1} \\
\sigma_{D 2} \\
\sigma_{D 3}
\end{array}\right\}=\left\{\begin{array}{l}
\varepsilon_{D 1} \\
\varepsilon_{D 2} \\
\varepsilon_{D 3}
\end{array}\right\}
\end{gathered}
$$

The quantities $\sigma_{\mathrm{Si}}$ and $\sigma_{\mathrm{Di}}$ are principal residual stresses sum $\left(\sigma_{\mathrm{Si}}=\sigma_{1 \mathrm{Ri}}+\sigma_{2 \mathrm{Ri}}\right)$ and difference $\left(\sigma_{D i}=\sigma_{1 R i}-\sigma_{2 R i}\right)$ for each layer at a given depth $h_{i}$. 


\section{Materials and Experimental Procedure}

Table 1 shows the mechanical properties of the test materials used in this investigation. Round geometrical specimens, with the geometrical dimensions shown in Fig. 6, have been subjected to a similar shot-peening treatment. Table 2 shows the shot-peening parameters used.

Table 1 Mechanical properties of the test materials

\begin{tabular}{|c|c|c|c|c|c|c|c|c|c|c|}
\hline \multirow{2}{*}{$\begin{array}{c}\text { Material } \\
\text { DIN }\end{array}$} & \multirow{2}{*}{$\begin{array}{c}\sigma_{\mathrm{ys}} \\
(0.2 \%) \\
{[\mathrm{MPa}]}\end{array}$} & \multirow{2}{*}{$\begin{array}{c}\sigma_{U T} \\
{[\mathrm{MPa}]}\end{array}$} & \multirow{2}{*}{$\mathrm{n}_{\mathrm{p}} *$} & \multirow{2}{*}{$\begin{array}{l}* * \\
\mathrm{HV}\end{array}$} & \multicolumn{6}{|c|}{ Chemical composition [\% weight] } \\
\hline & & & & & $\mathrm{C}$ & $\mathrm{Si}$ & $\mathrm{Mn}$ & $\mathrm{Cr}$ & Mo & $\mathrm{Ni}$ \\
\hline $14 \mathrm{Ni} \mathrm{Cr} 14$ & 460 & 590 & 0.14 & 210 & 0.14 & $<0.4$ & 0.55 & 0.75 & - & 3.25 \\
\hline $34 \mathrm{Ni} \mathrm{Cr}$ Mo 6 & 920 & 1000 & 0.06 & 340 & 0.34 & $<0.4$ & 0.65 & 1.50 & 0.22 & 1.50 \\
\hline
\end{tabular}

(*) Strain hardening exponent

(**) Vickers microhardeness of the bulk material

Table 2 Shot-peening parameters according to MIL-S-13165C standard

\begin{tabular}{cccc}
\hline Peening medium & Impact angle & Almen intensity & Coverage \\
\hline S170 & $\pm 90^{\circ}$ & $14 \mathrm{~A}$ & $100 \%$ \\
\hline
\end{tabular}
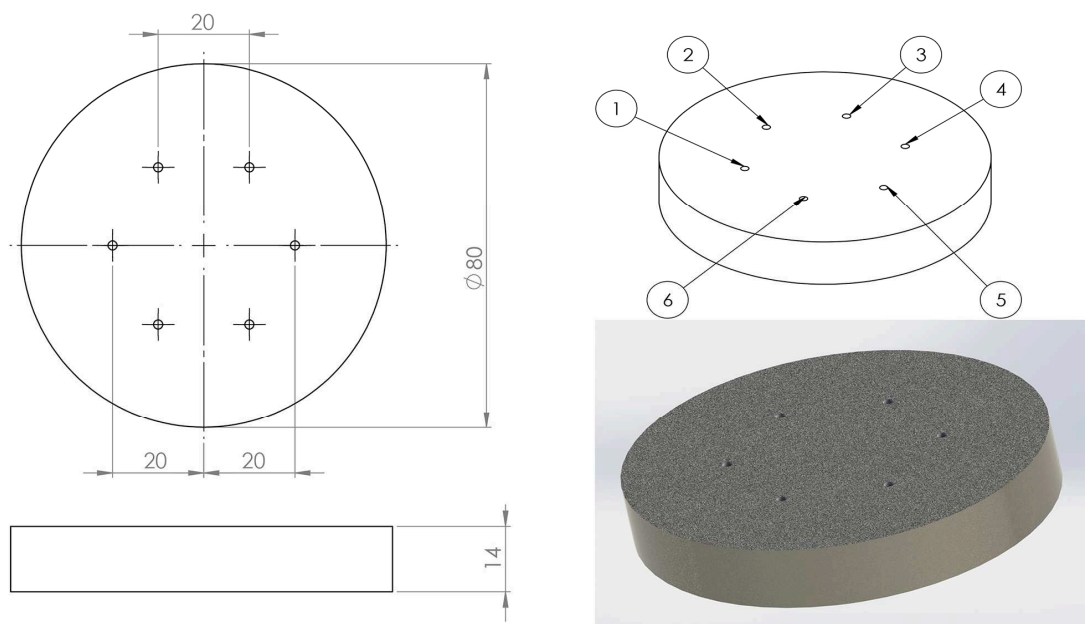

Fig. 6 Geometry of the specimens and measuring points

In each specimen, six measuring points have been considered. Three alternating points for IHD assisted by strain gauge technology $(1,3,5)$ and other three alternating points for IHD assisted by DSPI technology $(2,4,6)$. For comparison purposes, the central region of each specimen has been used to determine the residual stress depth distribution using the X-ray diffraction (XRD) $\sin ^{2} \psi$ method [5], selected as reference technique. XRD residual stress analysis was combined with the electrolytic layer removal technique to achieve residual stress depth profiles. Stress relaxation due to layer removal was not taken into account since the affected region was small and no significant relaxation effects could be expected. Lattice deformations of the Fe $\{211\}$ planes were determined on a conventional $\psi$ diffractometer for $15 \psi$ angles between $-45^{\circ}$ and $+45^{\circ}$ using $\mathrm{CrK} \alpha$ radiation. Residual stresses were calculated for the plain stress conditions using X-ray elastic constants of $1 / 2 \mathrm{~s}_{2}=5.832 \times 10^{6} \mathrm{MPa}^{-1}$ and $\mathrm{s}_{1}=-1.272 \times 10^{6} \mathrm{MPa}^{-1}$.

For the IHD technique assisted with strain gauge technology, high speed drilling equipment (milling guide RS-200 ${ }^{\circledR}$ with air turbine, Vishay Precision Group, Inc.) was used to perform a cylindrical blind hole. This technique is recommended since only minor drilling-induced plastic deformations occur and a satisfying hole shape can be achieved [6]. Surface strain relief was measured by a three-element strain gauge rosette (CEA-06-062UM-120 ${ }^{\circledR}$ ), Vishay Precision Group, Inc.), gauges being radially oriented around the hole at different angular positions. In depth increments of 0.02-0.08 mm, strain relief was measured after each depth increment to about $1 \mathrm{~mm}$ below the surface. Smaller steps were carried out close to the surface to access stress gradients more accurately. The typical hole diameter was about $1.8 \mathrm{~mm}$. For the residual stress evaluation the 
elastic constants $\mathrm{E}=210000 \mathrm{MPa}$ and $v=0.3$ were used. A similar procedure was used for the IHD technique assisted with DSPI technology. In this case, however, new high speed drilling equipment with the DSPI technology assembled on it, developed by the Labmetro [1], has been used.

\section{Experimental Results and Discussion}

Residual stresses as function of the depth were determined in both shot-peened steels. Fig. 7 shows the mean curve of the strain relaxation as a function of depth, corresponding to the three measurement points, determined using electric strain gauges (SG) and digital speckle pattern interferometry (DSPI). A clear difference can be observed for both steels. The strain relaxations curves determine by DSPI are always below those determined by SG. Since the residual stresses are related with the strain-depth gradients, it is possible to conclude that DSPI seems to underestimate the residual stress distribution existing in each shot-peened material. It is clear that both techniques present similar values of positive strain relaxation measured in three different directions, by the three strain gauges, indicating an equi-biaxial stress state induced by the shot-peening treatment. Fig. 8 only considers the value of the maximum principal residual stress.
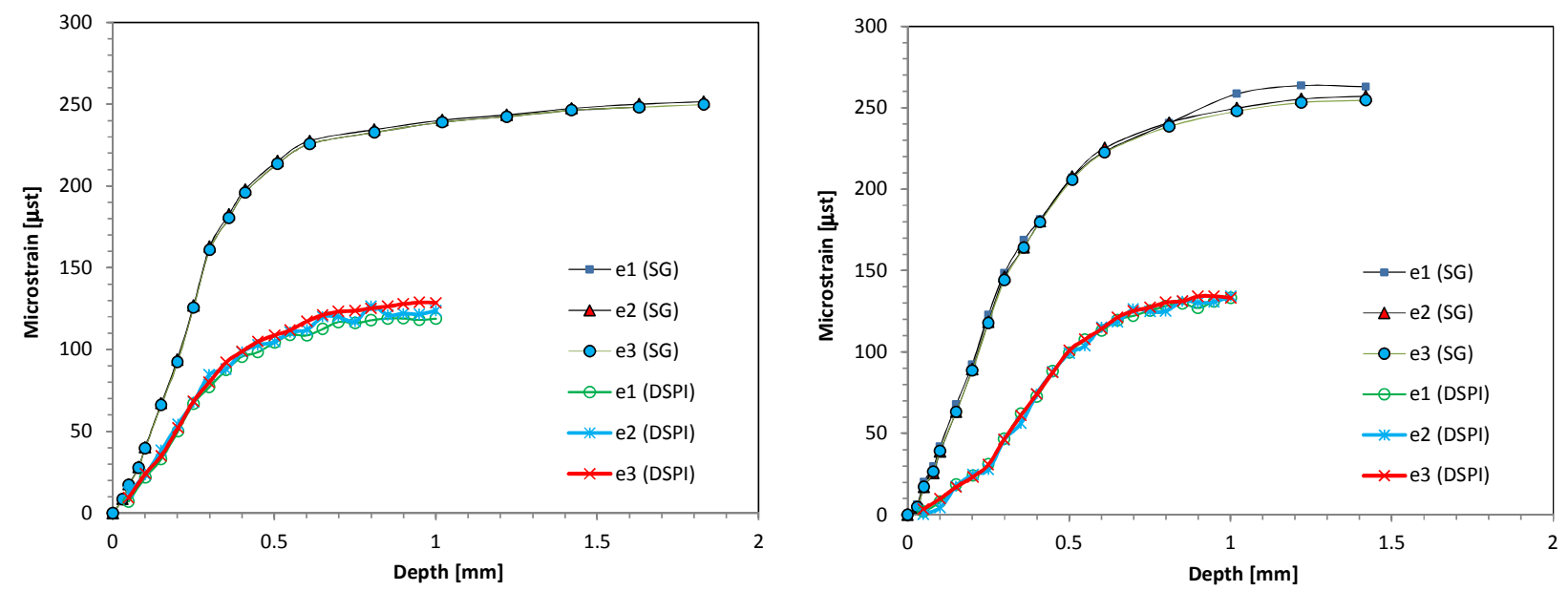

Fig. 7 Strain relaxation vs. depth distribution in shot-peened specimens DIN 34 Ni Cr Mo 6 (left) and DIN 14 Ni Cr 14 (right)
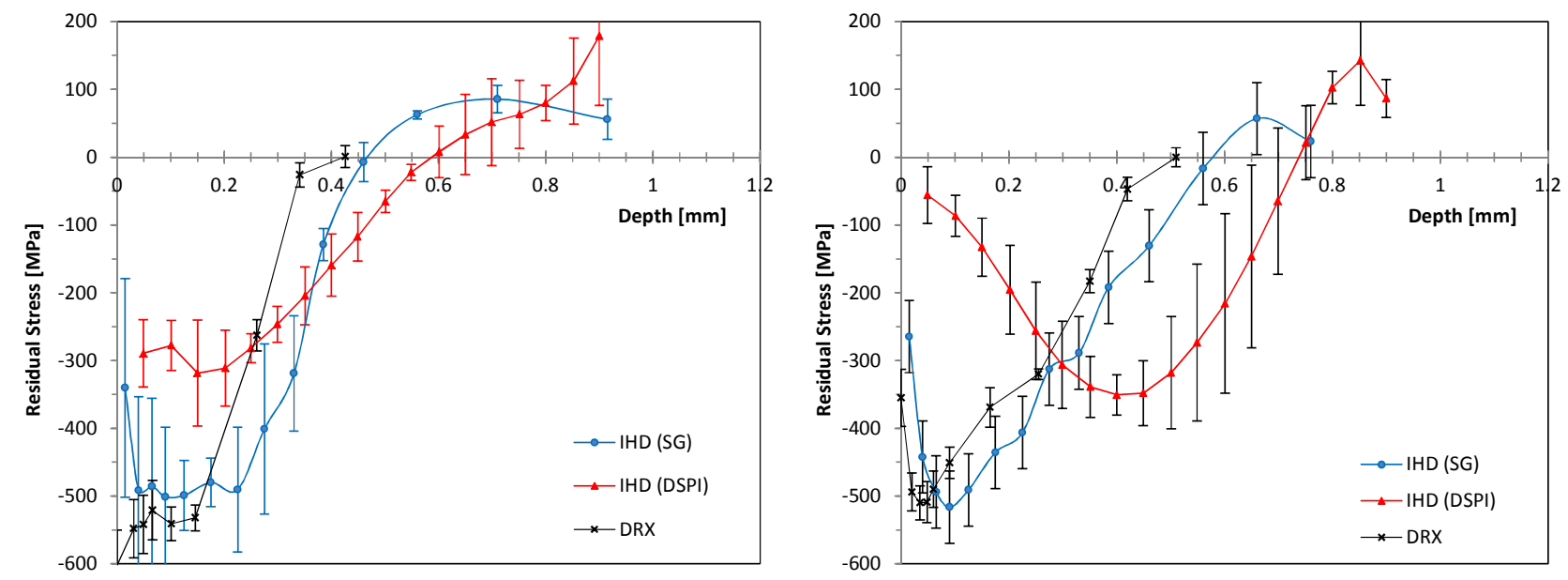

Fig. 8 Residual stress depth distribution in shot-peened specimens DIN 34 Ni Cr Mo 6 (left) and DIN 14 Ni Cr 14 (right)

Applying the integral method for residual stresses determination, according to the standard ASTM E-837-13a [3], it is possible to determine the shot-peening residual stresses induced in both materials, based on strain relaxation curves determined by SG and DSPI presented in Fig. 7. The residual stress results are presented in Fig. 8, and the curves correspond to the mean values of the 
maximum principal residual stress determined by both techniques in the three measurement points analyzed. Standard deviation is shown by the error bars in all curves. As expected, a discrepancy between the residual stress results, using SG and DSPI, can be observed, especially for the case of DIN 14 Ni Cr 14 specimens shown in Fig. 8 (right). For DIN 34 Ni Cr Mo 6 specimen, the residual stress results obtained by using the both strain measurement techniques are in better agreement. For comparison purposes and reference, the results determined by X-ray diffraction (XRD) are also shown in Fig. 8. As it can be seen, the residual stress results determined by the incremental holedrilling technique (IHD), using SG, approach those determined by X-ray diffraction, while a clear discrepancy is observed when IHD residual stress results, using DSPI, are compared with the residual stress results determined by XRD.

\section{Conclusions}

The systematic comparison between the shot-peened residual stresses determined by the incremental hole-drilling technique (IHD), using standard electric strain-gauges (SG) and digital speckle pattern interferometry (DSPI), leads to the conclusion that the enhanced digital speckle pattern interferometry technique for measuring the strain relaxation arising around the drilled holes and, consequently, the in-depth residual stress distribution induced by shot-peening, did not present the expected accuracy. As reference, the X-ray diffraction technique (XRD) was combined with the electrolytic layer removal technique to determine the shot-peening residual stress depth profiles in all specimens. The residual stresses determined by IHD, combined with SG, are in good agreement with those determined by XRD, while clear discrepancies were observed between these values and those determined by IHD, combined with DSPI. Therefore, the improvement of the accuracy of DSPI for measuring strain relaxation values during IHD should still be pursued in the future.

\section{References}

[1] Albertazzi A, Kanda C, Borges MR, Hrebabetzky F. Portable residual stresses measurement device using ESPI and a radial in-plane interferometer. Proc. SPIE 4420, Laser Metrology for Precision Measurement and Inspection in Industry. A. Albertazzi ed. Florianópolis, Brazil, SPIE, 2001.

[2] Viotti MR, Albertazzi A. Compact sensor combining digital speckle pattern interferometry and the hole-drilling technique to measure nonuniform residual stress fields. Optical Engineering (SPIE). 52 (2013) 101905-12.

[3] ASTM-E-837-13a. Standard Test Method for Determining Residual Stresses by the HolleDrilling Strain-Gage Method. Repprinted from Annual Book of ASTM Standards. 2013: 1-16.

[4] Schajer GS. Measurement of Non-Uniform Residual Stress Using the Hole-Drilling Method. Part II-Practical Application of The Integral Method. Journal of Eng. Mat. and Tech. (ASME). 110 (1988) 344-349.

[5] Macherauch E, Müller P. Das sin2y-Verfahren der Röntgenographischen Spannungsmessung. Zeitschrift für Angewandte Physik. 13 (1961) 305-317.

[6] Flaman MT. Brief investigation of induced drilling stresses in the center-hole method of residual-stress measurement. Experimental Mechanics. 22 (1982) 26-30. 